\title{
COLOR AND TEXTURE BASED IMAGE RETRIEVAL: A PROPOSED METHOD
}

\author{
Avneet Kaur ${ }^{1}$, Vijay Kumar Banga ${ }^{2}$, Navkirat Kaur ${ }^{3}$ \\ ${ }^{1,2,3}$ Amritsar College of Engineering \& Technology, Amritsar, India
}

\begin{abstract}
Content-based image retrieval (CBIR) is an vital research area for manipulating bulky image databases and records. Alongside the conventional method where the images are searched on the basis of words, CBIR system uses visual contents to retrieve the images. In content based image retrieval systems texture and color features have been the primal descriptors. We use HSV color information and mean of the image as texture information. The performance of proposed scheme is calculated on the basis of precision, recall and accuracy. As an effect, the blend of color and texture features of the image provides strong feature set for image retrieval.
\end{abstract}

Keywords: image retrieval, HSV color space, color histogram, image texture.

\section{INTRODUCTION}

A preliminary approach for organizing large image collections is to use keywords that refer to properties of the image, which may include the theme, the position, or the time of the image. The image retrieval structure which is based on words or text is identified as text-based image retrieval or metadata-based image retrieval [2]. This approach has some obvious shortcomings. Different people may classify or express the same image in a different way, leading to troubles retrieving it again. When concerning with bulky databases it consumes more time. [4]

Text-based image retrieval schemes utilize text to illustrate the content of the image that stimulates uncertainty and insufficiency in image database search and query processing. This trouble is due to the complexity in specifying accurate language and phrases in unfolding the content of images as it is much complex than what any set of keywords can convey. Since the textual remarks are based on language, which might fluctuate according to every user, therefore variations in notation will create challenges to image retrieval [3]. Brahmi et al. mentioned the following two drawbacks in text-based image retrieval. First, manual image explanation is time-consuming and precious as well. Second, human annotation is immanent. Also, Sclaroff et al. pointed that various images could not be explained with word or set of words because it is hard to express their content with terminology [5]. Thus, in text based image retrieval, possibility of error is more. This gives rise to a new technique for image retrieval which recovers images on the basis of contents of the image and so called content based image retrieval system.

\subsection{Color Based Image Retrieval}

There are so many techniques to extract relevant images on the color basis. Every image in the database is processed to determine its color histogram which depicts the quantity of pixels of each color contained by the image. Then these color histograms are saved in the record. During the process, the end user can also give the desired percentage of each color (for example $86 \%$ green and $14 \%$ blue) or load an example image as a query whose color histogram is computed. Then, the similarity measure searches those images from database whose color histograms resembles strongly with the query image. Swain and Ballard was first introduced a matching method called histogram intersection which is most commonly used [1991]. The use of cumulative color histograms is considered as improvement to Swain and Ballard's original technique. The combination of histogram intersection with several aspect of spatial matching, and region-based color query, is also very popular. The results generated from some of these methods look quite imposing.

\subsection{Texture Based Image Retrieval}

The retrieval of images on the texture basis might not appear very practical. However the ability to match on texture comparison can be helpful in distinguishing between areas of images with similar color. A range of methods has been used for measuring texture similarity. With the help

of these it is feasible to compute image texture which includes the level of contrast, unevenness, directionality and regularity, or periodicity plus uncertainty. The use of Gabor filters and fractals are the techniques of texture examination for image retrieval. Texture queries can be submitted in an analogous approach to color queries, either by choosing examples of required textures or by providing a image. The 
scheme then retrieves images with texture measures most close match in accordance to the query. A fresh addition of the method is the texture lexicon, which recover textured regions from images on the basis of similarity to automatically-derived cipher words on behalf of significant classes of texture within the image collection.

\section{PROPOSED ALGORITHM}

Our new approach deals directly with those applications in which user require a specific image of our interest from a large image database. In the paper, we have planned a strong content-based image retrieval process based on an proficient blend of color and texture features. Both color and texture features of images are extracted and saved as feature vectors in a database. As in any content based image retrieval, there are some steps to be followed.

Similarly, in this method a query image also has to undergoing through these steps. During this retrieval procedure, the color and texture element vector of the query image is calculated and compared against those features stored in the database.

\section{IMAGE RETRIEVAL PERFORMED WITH}

\section{THE HISTOGRAM VALUE AND TEXTURE DESCRIPTOR}

In some situations collective value of color and texture feature works very well. This algorithm is also based on the same theory as it utilizes histogram of hsv image and mean of the image. The whole process that occurred in purposed work basically involves two levels:

1) Color based image retrieval.

2) Texture based image retrieval.

As shown in Figure 1 when a user load the query image, its undergoing through level 1 which is color based image retrieval. Its color features are selected and matching process is carried out between query image features and the images features that are stored in the database, the results closes to the query image is then retrieved from the database and displayed as the output of level 1 . The next step is to extract texture feature of query image as well as the images retrieved from previous level. Then the matching similarity and indexing of images against query image has done. The images relevant to the query image is then retrieved and displayed.

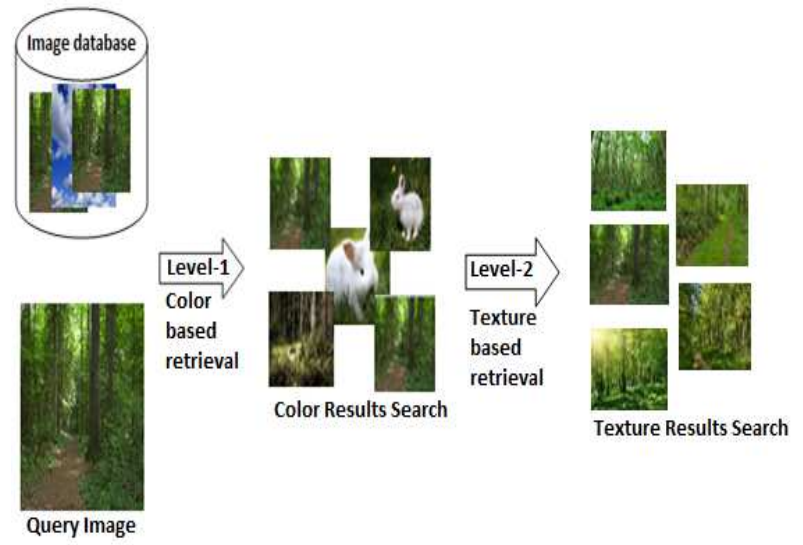

Fig 1: Two-level proposed system

\subsection{Procedure for Color Feature}

We evaluate HSV color space of the images that are stored as database for purposed content based image retrieval. HSV symbolizes Hue, Saturation and Value, provides the perception illustration according with human being visual quality.

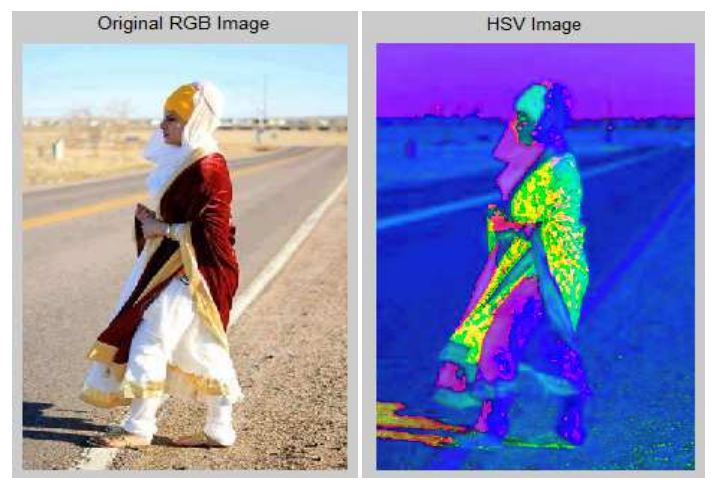

Fig 2: Original RGB Image \& Image in HSV domain

Step 1: Load database in the MATLAB workspace.

Step 2: Convert RGB image into HSV.

Step 3: Produce histogram of HSV image.

Step 4: Then the number of bins in each direction is duplicated by means of interpolation.

Step 5: Store HSV values of database images into a mat file.

Step 6: Input the query image.

Step 7: Apply step 2-4 to get HSV values of query image.

Step 8: Find out the Euclidean distance of query image and stored database images.

Step 9: Keep only distances which are larger than predefined threshold (say T1).

Step 10: find the distance values which are smaller than T2 (another predefined threshold).

Step 11: Compute similarity measure using mean and length of the two threshold applied output.

Step 12: Sort the similarity values to perform indexing 
Step 13: Plot the Similar Images and keep first 60-70 images for further processing.

\subsection{Procedure for Texture Feature}

For texture based image retrieval, database is the result of previous level. Mean of these images are computed and compared against the mean of query image. The various steps during this retrieval process are as follows:

Step1: Take the result of color based retrieval as the database. Step 2: Resize the images for [256, 256].

Step 3: Find mean of each image.

Step 4: Submit query image and apply step $2 \& 3$ to get its mean.

Step 5: Find out the Euclidean distance of query image and database images.

Step 6: Keep only distances which are larger than predefined threshold (say T1).

Step 7: Find the distance values which are smaller than T2 (another predefined threshold).

Step 8: Compute similarity measure using mean and length of the two threshold applied output.

Step 9: Sort the similarity values to perform indexing.

Step 10: Display 1-20 results on GUI.

\section{RESULTS:}

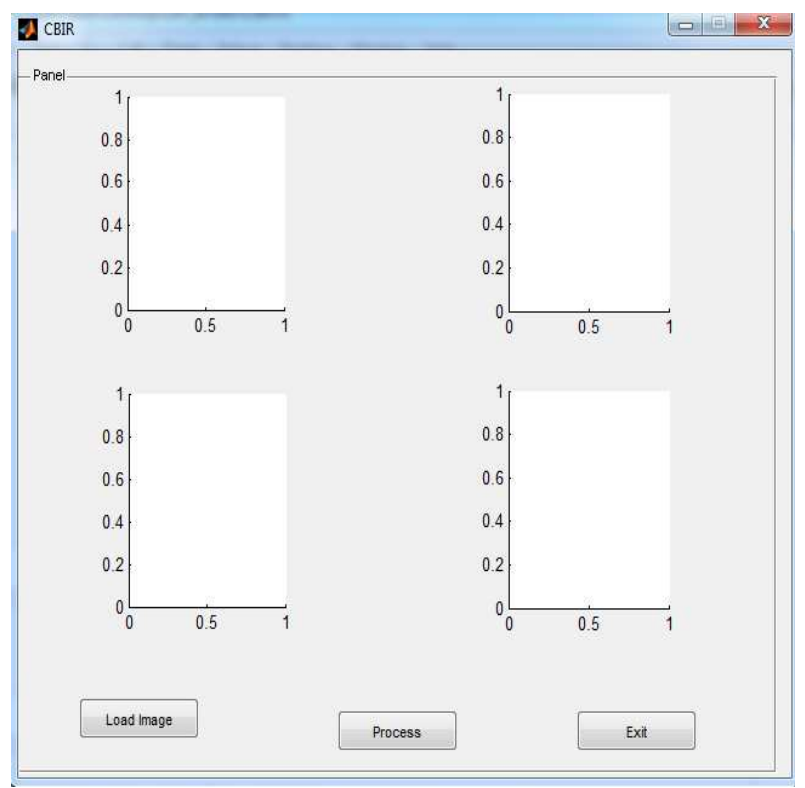

Fig 3: Structured GUI for proposed work

1. Input Query image using 'Load Image'.

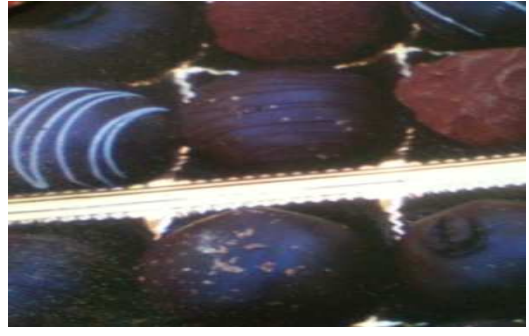

Fig 4: Example of Query Image

2 Histograms are plotted as per below figure

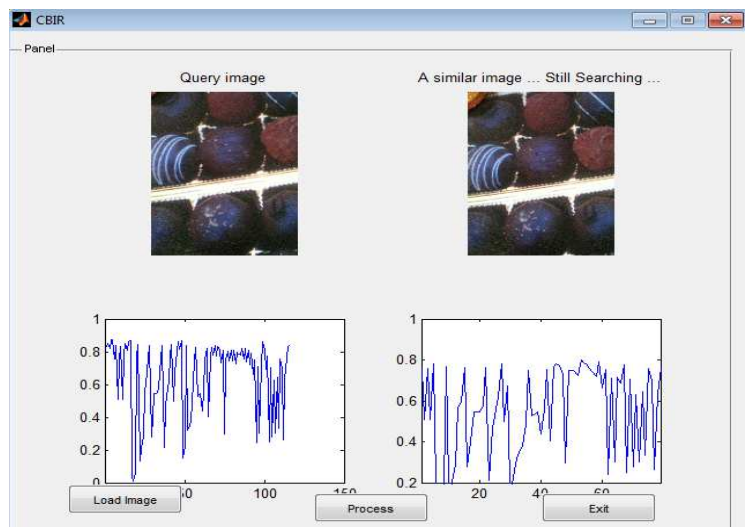

Fig 5: Processing of retrieval system

3. All the images in database that are matched in terms of color are displayed below

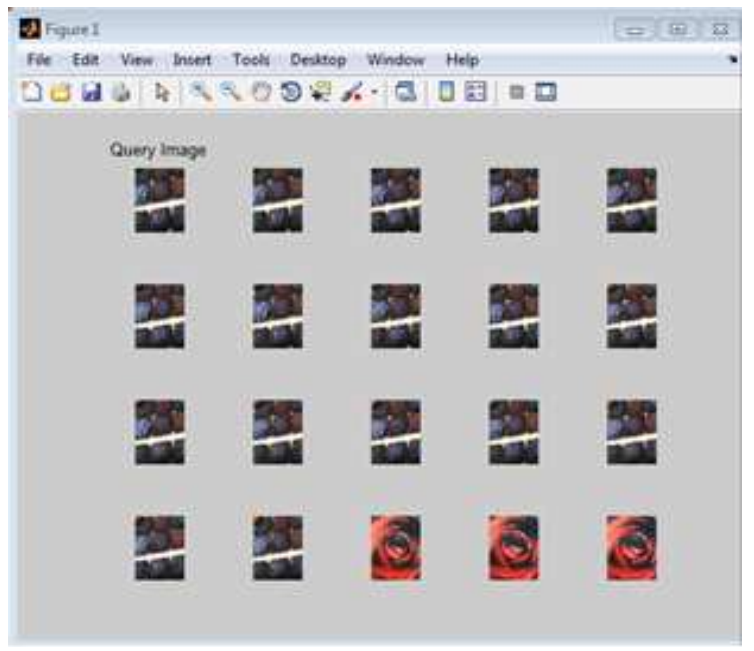

Fig 6: Color search results

4. After Completion of Color retrieval, the resulted images are processed for texture analysis.

5. Texture Extraction is done for only images shown in the above figure. 


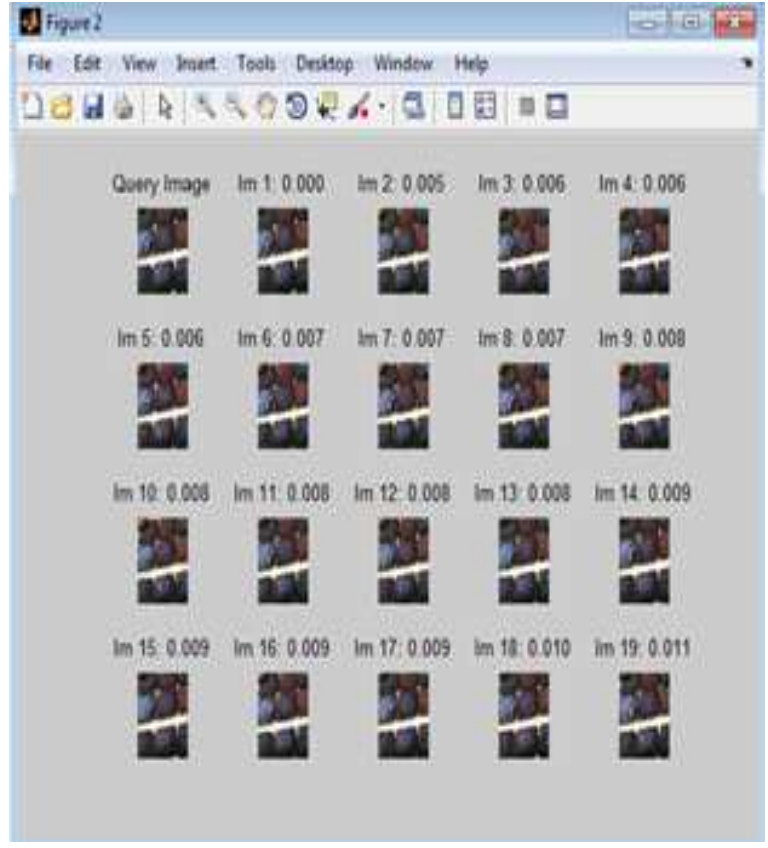

Fig 7: Results of proposed scheme retrieval system

\section{PERFORMANCE EVALUATION:}

The performance of any retrieval system is measured by computing recall and precision. Recall determine the capability of the method to recover all the models that are appropriate, whereas precision compute the ability of the method to retrieve just models that are pertinent [1]. They are expressed as:

$$
\begin{gathered}
\text { Precision }=\frac{\text { No. of Relevant Images Retrieved }}{\text { Total no. of Images Retrieved }} \\
\text { Recall }=\frac{\text { No.of Relevant Images Retrieved }}{\text { No.of Relevant Images in the database }}
\end{gathered}
$$

After calculating Precision and Recall, Accuracy is calculated, this is the average value of Precision and Recall.

\section{CONCLUSIONS}

The paper proposed a method for image retrieval using hsv histogram values and texture descriptor analysis of image. We first convert a true color image into hsv image. We build up a method for image retrieval which is based on the histogram values. After extraction of color feature, texture feature is extracted from image. When query image is submitted, its color and texture value is computed which is then matched against color and texture values of various images saved in database. The images whose value is similar to query image are then retrieved and displayed on GUI as result. The presented method provides low computational complexity and high retrieval accuracy.

\section{REFERENCES:}

[1] Ding-Yun Chen, Xiao-Pei Tian, Yu-Te Shen and Ming Ouhyoung, "On Visual Similarity Based 3D Model Retrieval", EUROGRAPHICS 2003, Association and Blackwell Publishers 2003. Volume 22 (2003), Number 3 [2] Eustratios Moutousidis, Michael Vassilakopoulos, "An Image Database System supporting Retrieval by Content", [3] Hui Hui Wang, Dzulkifli Mohamad, N.A Ismail, "Image Retrieval: Techniques, Challenge, and Trend", World Academy of Science, Engineering and Technology 602009 [4] Mr. Milind V.Lande, Prof. Praveen Bhanodiya, Mr. Pritesh Jain, "Efficient Content Based Image Retrieval Using Color and Texture", International Journal of Scientific \& Engineering Research, Volume 4, Issue 6, June-2013 121

[5] Neetu Sharma, Paresh Rawat and Jaikaran Singh, "Efficient CBIR Using Color Histogram Processing", Signal \& Image Processing : An International Journal(SIPIJ) Vol.2, No.1, March 2011 